\title{
In silico study to examine the role of amino acids in Jasmonate induced plant defense process
}

\section{Ruma Ganguly and Sailesh K. Mehta*}

Sir P. P. Institute of Science. M. K. Bhavnagar University. Bhavnagar. Gujarat. India. *Email: skmehta40@rediffmail.com.

\begin{abstract}
The role of amino acid is important to Jasmonate induce plant defense process. Jasmonic acid and amino acid Isoleucine conjugate (JA-Ile) has been found to be necessary to achieve such process effectively. We have examined the origin of such process computationally and showed that Isoleucine is more active compared to other Jasmonic acid conjugates. The epimerization process revealed that Isoleucine conjugated Jasmonic acid is energetically a favoured process compared to JA-Leu and JA-Val. Water has functioned as a catalyst in the whole epimerization process. This study would unravel the importance of Isoleucine in the Jasmonic acid induced plant defense process.
\end{abstract}

Keywords: Jasmonic acid; JA-Ile; JA-Leu; JA-Val; Enolization; Planarized structure.

\section{Introduction}

The plants are exposed to various biotic stresses, mainly insect attacks, on earth. They have to compete every day to sustain in the earth's incompatible environment. As plants have to defend themselves from these insect attacks on the spot and have to produce some sort of defense to check further attacks, they have evolved a variety of elicitors as a defense mechanism to deter herbivores
Received

May 30, 2018

Accepted

August 24, 2018

Released

August 31, 2018

Full Text Article

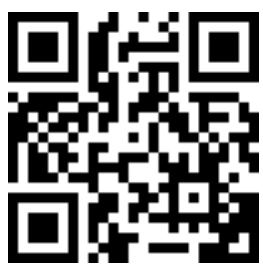

D 0000-0002-8897-3610

Ruma Ganguly

(D) 0000-0002-5044-5629

Sailesh K. Mehta specially the plant eating insects (Gundlach et al.,1992; McConn et al., 1997). One important elicitor in that group is Jasmonic acid and its derivatives collectively called as 'Jasmonates' Jasmonates act as signaling molecules which trigger the expression of defense related genes (Ryan, 1992; Koo, 2009). Biosynthesis of Jasmonic acid takes place from a polyunsaturated fatty acid called $\alpha$-linolenic acid. with various enzymatic actions and three round of $\beta$-oxidations 
(Vick and Zimmerman,1984; Browse et al., 2008; Stintzi et al., 2002). The final product

(+)-7-iso-JA(3R,7S) subsequently equilibrates to the more stable trans-Jasmonic acid (3R,7R) or (-)JA(3R,7R) (Wasternack, 2007, 2010; Feussner and Wasternack, 2002). However, it is not the (+)-7-iso-Jasmonic acid (3R,7S) which is directly involved in the defence mechanism in plants. Activation of Jasmonic acid with the conjugation with the amino acid is necessary before the actual defense process begins. The active derivative of Jasmonic acid involved in defence mechanism in plants reported is the amino acid Isoleucine conjugate of Jasmonic acid, i.e. (+)-7-iso-JA-LIle(3R,7S) which interacts with the receptor COI1 and helps to bind to JAZ protein (Fonseca et al., 2009). In plant there are other two amino acids present which have structural similarity with Isoleucine and are reported to form conjugate with Jasmonic acid. These amino acids are Leucine (Leu) and Valine (Val) (Piotrowska and Bajguz, 2011). All the three amino acids i.e Valine, Leucine and Isoleucine are non-polar aliphatic amino acids with an $\alpha$-amino acid group, an $\alpha$-carboxylic group and different branched side chains. Isoleucine contains a methyl substituted alkyl side chain, in Leucine the side chain is comprised of isobutyl group and in Valine the side chain is of isopropyl group. According to earlier reports, although JA-L-Ile is the highly reactive conjugate but JA-L-Leu and JA-L-Val conjugates too have shown weak activity (Miersch et al., 1999; Wasternack, 2010; Piotrowska and Bajguz, 2011; Naoki et al., 2014).

This article examines the activity of these amino acids towards the plant defense mechanism computationally. The experimental observations report the relative activity of such amino acid conjugated jasmonates, however, the origin of such activity is not well explored. The biological activity of such amino acid conjugates assumed to appear from the peculiar structural feature of Jasmonic acid during its interaction with the macromolecular host (Krumm et al., 1995). The experimental data support that Jasmonic acid enolizes during or before its interaction with the appropriate functional group of receptor molecule. In the enolization process, the sp2 carbon center is created in the cyclopentanone ring, which planarize the upper half of the jasmonate molecule. The role of the hydroxy group (or the enol form of Jasmonic acid) is considered to be important for the formation of hydrogen bond with an appropriate functional group at the receptor side. It appears that directionality and steric constraints are exerted by the planar enolized segment of the jasmonate molecule for successful signal transduction (Krumm et al., 1995). Further, the role of stereoisomeric forms of amino acid conjugated Jasmonic acid is also examined.

\section{Computational methods}

All geometry optimizations have been carried out with Semi-empirical AM1 method (Dewar et al., 1985). The transition state geometries were also found out with AM1 and characterized by single imaginary frequency calculations. All minimum were characterized with positive frequencies in the frequency calculations. B3LYP/6-31+G* level of theory employed to calculate the single point energies of the systems studied and reported here (Hehre et al., 1986; Lee et al., 1988; Becke, 1992). Solvent model calculations were carried out with AM1SM2 solvation model (Dewar et al., 1985; Cramer and Truhlar, 1992). All calculations were carried out with SPARTAN programme (Dewar, 1985).

\section{Result and discussions}

Fonseca et al. (2009) have shown that the endogenous bioactive jasmonate in neutral $\mathrm{pH}$ conditions is (+)-7-iso-JAL-Isoleucine, however, is known to be 
less stable than the corresponding transderivative(-)-JA-L-Ile (Miersch et al., $1999 \mathrm{~b}$ ). It has been reported that epimerization leads to the production of the more stable trans derivative (Fonseca et al., 2009). Wasternack et al. (2009) further reported that the similar derivatives, i.e. (-)-JA-L-Val and (-)-JA-LLeu, are weakly active compared to (+)7-iso-JA-L-Isoleucine. Jasmonic acid possess two chiral centers, $\mathrm{C}-3$ and $\mathrm{C}-7$ positions (Figura 1) at cyclopentanone ring (Holbrook et al., 1997). The chiral center C-7can participate in epimerizaton as the $7 \mathrm{CH}$ hydrogen is next to the carbonyl group and it is believed that it undergoes a keto-enol tautomerization and undergoes epimerization at $\mathrm{C}-7$ to form the trans stereoisomer, while C-3 does not take part in epimerisation and remains unchanged (Holbrook et al., 1997). The epimerization process for the amino acid conjugate of Jasmonic acid is not explored in the literature.

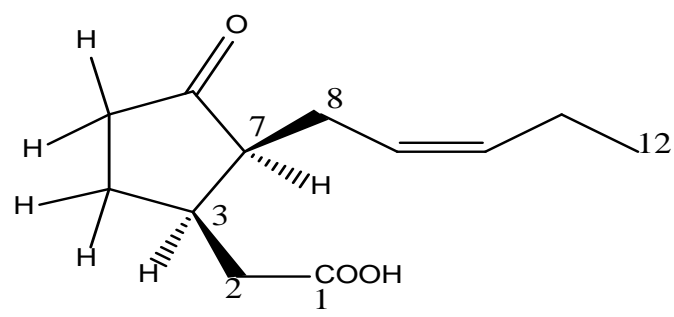

Figura 1. Structure of Jasmonic acid.

The structural and energetics to undergo from (+)-7-iso-JA-L-Isoleucine to trans-derivative (-)-JA-L-Ile needs attention. Further, the structurally similar Valine and Leucine conjugated with Jasmonic acid is feeble in activity compared to Isoleucine, also needs to be addressed. It is known in the literature that acid, base participates in the ketoenol tautomerization process to accelerate the formation of enol form (Krumm et al., 1995). In neutral conditions, water may catalyse the ketoenol tautomerization in plants, as water is available in sufficient quantity. Hence, one might anticipate that water can play a big role to epimerize the amino acid conjugated Jasmonic acid. We have performed quantum chemical calculations using semi-empirical AM1 and DFT methods to examine the potential energy surfaces of Isoleucine, Leucine and Valine conjugated to Jasmonic acid. These results would help to shed light on the relative efficacy of amino acid on plant defense mechanism. All the geometries considered have been optimized with AM1 level of theory in vacuum phase and further single point energy calculations were carried out with DFT calculations.

The epimerization process in JA-L-Ile(3R,7S) in presence of water molecule

The epimerization of (+)-7-isoJA-L-Isoleucine to its trans-derivative (-)-JA-L-Ile (3R,7R) has been examined with AM1 level of theory. The keto-enol tautomerization process to achieve the trans-form from the cis-form was carried out with a water molecule (Figure 2). It is known that the uncatalyzed keto-enol process is energetically much unfavourable compared to the catalysed process, hence the computations were performed with a water molecule (Dewar et al., 1985; Hehre et al., 1986; Becke, 1992; Lee et al., 1988). The carbonyl group of cis- Jasmonic acid-LIsoleucine $(3 \mathrm{R}, 7 \mathrm{~S})(\mathbf{1})$ interacts with the water molecule to transfer the hydrogen from the C-7 carbon atom of the cyclopentanone ring (Figura 1). The $\mathrm{C}=0$ takes the hydrogen from the water molecule in the transition state and the water takes the hydrogen from the C-7 atom (2). The enol-intermediate (3) shows that the water molecule is bound with the enol proton of cis-Jasmonic acidL-Isoleucine. In the enol formation, the double bond of $\mathrm{C}=0$ breaks down and hydroxyl $\mathrm{OH}$ - group is formed at C-6 position leading to the formation of a 


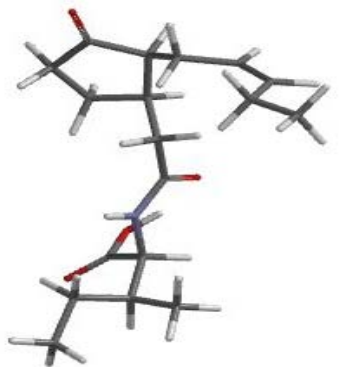

1

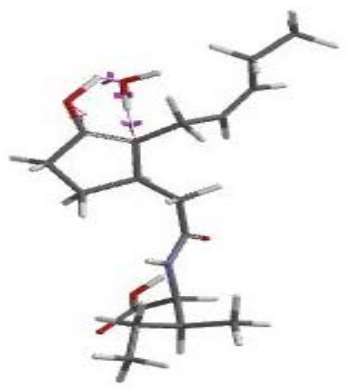

4

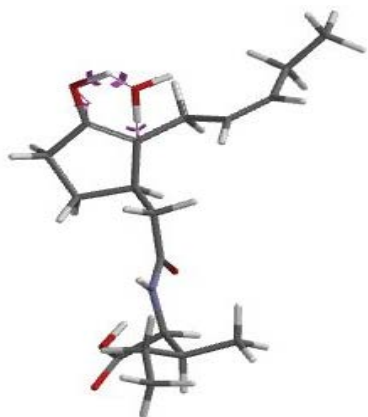

7

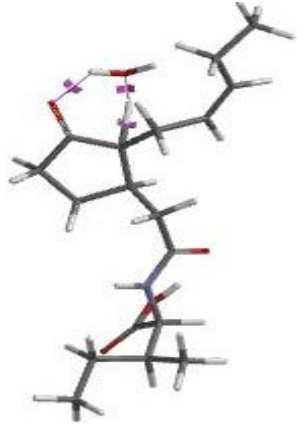

2

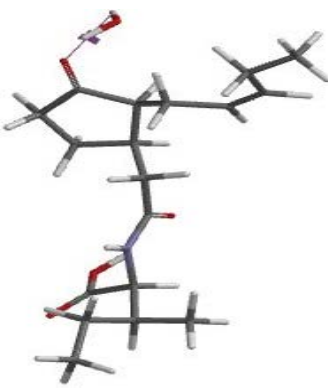

5

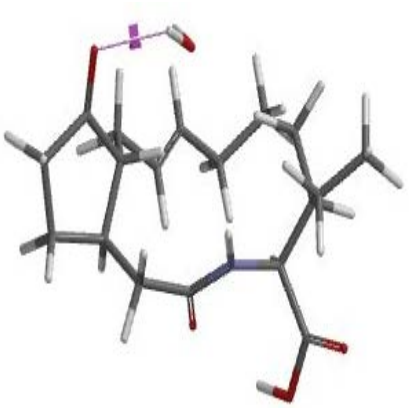

8

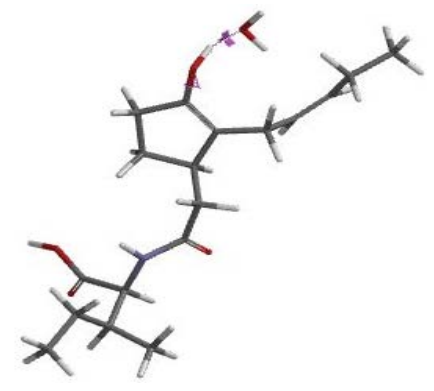

3

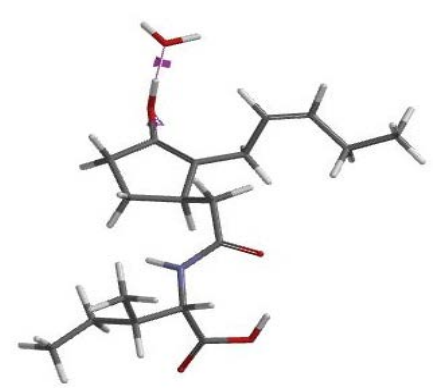

6

Figure 2. Geometry of JA-L-Ile $(3 \mathrm{R}, 7 \mathrm{~S})$ obtained by optimization in AM1 Semi empirical calculations. [1 = Start (cis-JA-L-Ile(3R,7S)), $\mathbf{2}$ = Transition State, $\mathbf{3}$ = Intermediate Enol form(syn-) $\mathbf{4}$ = Enol-Transition State (i), $\mathbf{5}=$ Final (cis-JA-L-Ile(3R,7S))attached to water, $\mathbf{6}=$ Intermediate Enol form (anti-), 7 = Enol-Transition State (ii), 8 = Final (trans-JA-L-Ile(3R,7R)) attached to water.

double bond between C- 6 atom and C-7 atom of cyclopentanone ring (Figure 2). The epimerization can possibly occur when the water molecule transfer the hydrogen to the C-7 carbon of the cyclopentanone ring. Such process can undergo in two different ways: (i) the water molecule delivers the hydrogen from the syn-side of the Isoleucine conjugated side chains (3) and (ii) if the water molecule orients away from this side chain(6). We have explored both possibilities in this case. The water molecule is hydrogen bonded to the enol- hydroxy group in both case (3 and $\mathbf{6}$ ). Further the water molecule can donate its hydrogen to the C- 6 carbon centre and the hydroxy hydrogen can be taken by the same water molecule. The transition state geometries were obtained for both (i) and (ii) (4 and 7 respectively). In enol-transition state (4), the enolic hydrogen is elongated by $1.6 \AA$, the distance between the enolic hydrogen atom and the oxygen atom of water molecule is $1.0 \AA$. The distance between of $\mathrm{C}-7$ atom of the cyclopentanone ring and hydrogen atom of the water 
molecule is $1.9 \AA$.Whereas, in enoltransition state (7) the enolic hydrogen is elongated by $1.5 \AA$, the distance between the enolic hydrogen atom and the oxygen atom of water molecule is
1.1 A. The distance between of C-7 atom of the cyclopentanone ring and hydrogen atom of the water molecule is $1.8 \AA$. It appears that the transition state (7) is much tighter than transition state (4).

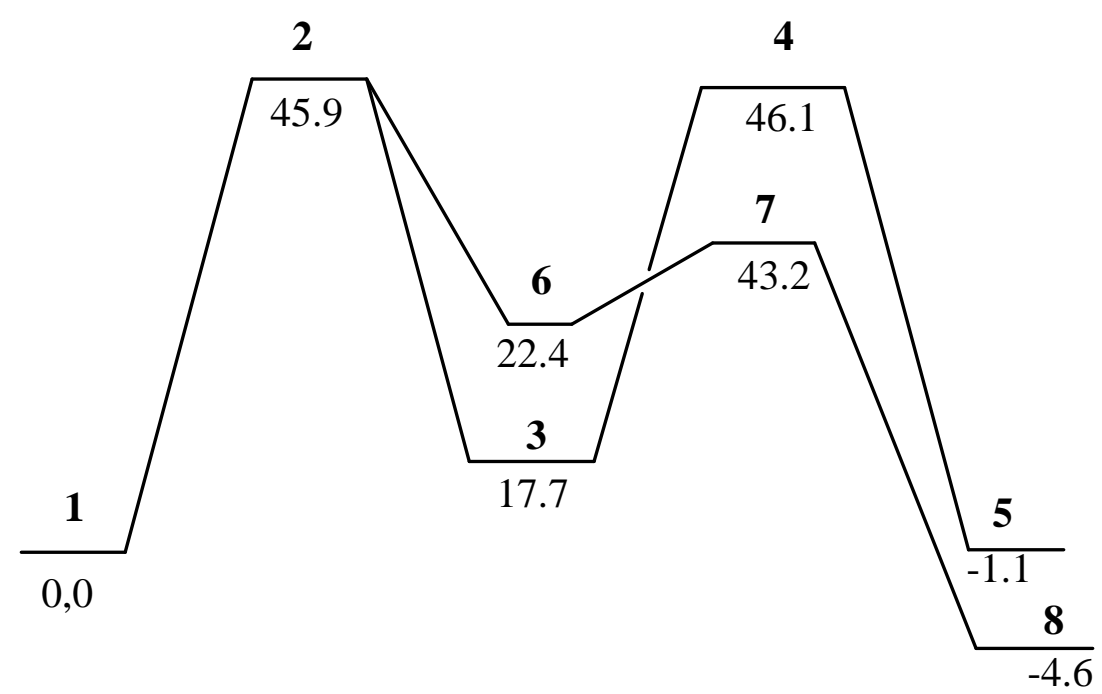

Figure 3. Potential energy surface profile for JA-L-Ile $(3 \mathrm{R}, 7 \mathrm{~S})$ in gas phase [All the energies are in $\mathrm{kcal} / \mathrm{mol}]$.

We have plotted the potential energy surface profile for the epimerization of $(+)$-7-iso-Jasmonoyl-LIsoleucine to its trans-derivative (-)-JA-LIle (3R,7R) (Figure 3) The optimized geometries at AM1 level of theory has been reported here however, the relative energies have been taken from the single point calculations performed with DFT level of theory. It is known that DFT level can predict more accurate energies compared to AM1 method (Cramer, C. J. and Truhlar, D. G.,1992). Epimerization of (+)-7-iso-JA-L-Isoleucine $(3 R, 7 S)$ to trans-derivative(-)-JA-L-Ile $(3 \mathrm{R}, 7 \mathrm{R})$ has been carried out with catalysing with one water molecule. The energies have been considered here in gas phase at DFT level of theory.The activation barrier calculated for the transfer of C-7 hydrogen to the carbonyl group of cyclopentaonone ring with water assistance (2) was found to 45.9
$\mathrm{Kcal} / \mathrm{mol}$ at B3LYP $/ 6-31+\mathrm{G}^{*}$ level of theory (Figure 3). The transition state (2) can possibly leads to two different enol intermediates, where the enol $\mathrm{OH}$ can be syn- to the 2-pentenyl side chain (3) and the $\mathrm{OH}$ anti- to the 2-pentenyl side chain (6). The enol intermediate (3) is relatively stable compared to (6) in DFT level of theory (Figure 3). The enol intermediates can further undergo to the formation of keto-forms assisted with a water molecule. The enol intermediate (6) proceeds with lower activation barrier compared to the enol intermediate (3). The activation barrier calculated using B3LYP/6-31+G* level is negative (Figure 3 ). The trans-derivative $(-)-J A-L-I l e(3 R, 7 R)(8)$ is energetically relatively stable compared to the corresponding (+)-7-iso-JA-L-isoleucine (5) (Figure 3). This clearly indicates that once reached to the intermediate enol stage, the system will favour the path (ii) 
leading to the second transition state (7). The formation of (8), i.e. the trans-form of Isoleucine conjugated Jasmonic acid is energetically favoured by $3.6 \mathrm{kcal} / \mathrm{mol}$ compared to the corresponding cis form
(5). The DFT results show that the formation of the trans-JA-L-Ile (3R,7R) will be the favoured as the stable product.

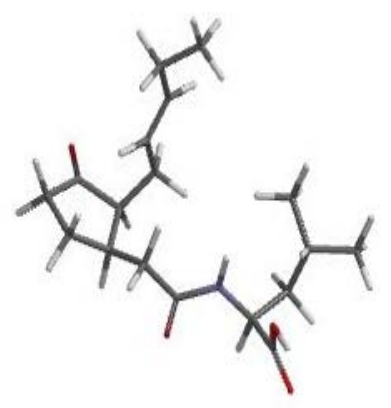

9

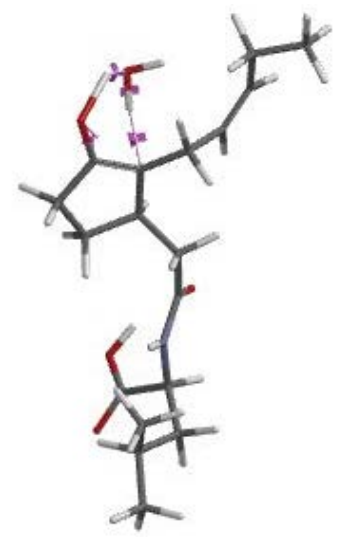

12

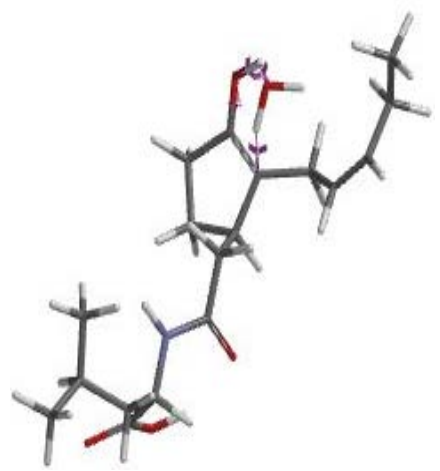

15

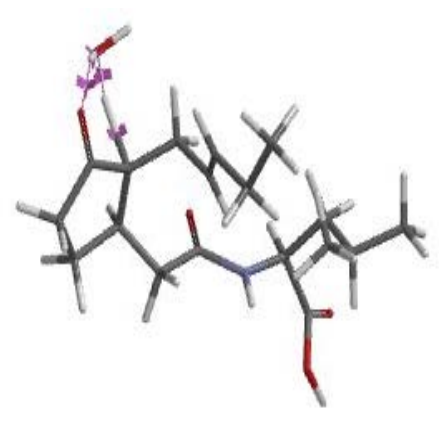

10

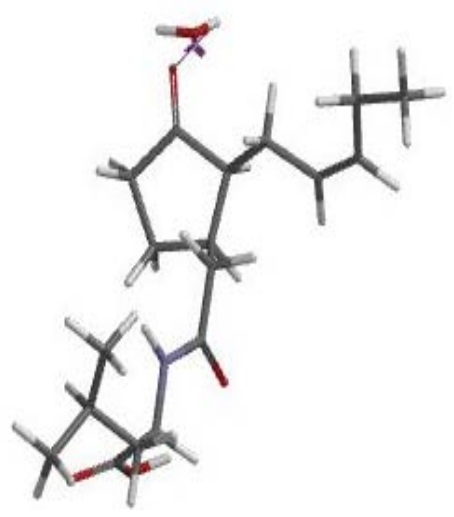

13

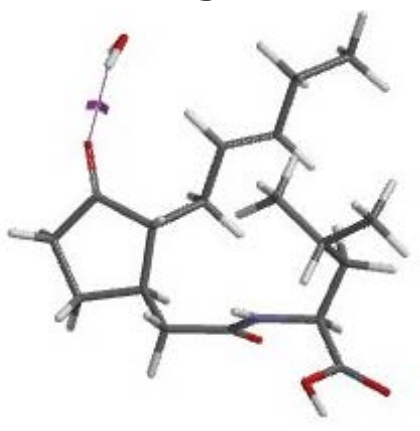

16

Figure 4. Geometry of JA-L-Leu $(3 R, 7 S)$ obtained by optimization in AM1 semi-empirical calculations. [9 = Start (cis- $(+)$ JA-L-Leu $(3 R, 7 S)$ ), $\mathbf{1 0}=$ Transition State, $\mathbf{1 1}=$ Intermediate Enol form (syn-), 12 = Enol-Transition State(i), 13 = Final $((+) J A-L-L e u(3 R, 7 S))$ attached to water, 14 = Intermediate Enol form (anti-), 15 = Enol-Transition State (ii), 16 = Final (trans(-)JA-L-Leu $(3 R, 7 R))$ attached to water]. 


\section{The epimerization process in JA-L-Leu $(3 R, 7 S)$ in presence of water molecule}

We have examined the epimerization of JA-L-Leucine to its trans-derivative JA-L-Leu(3R,7R) using AM1 and DFT levels of theory. The calculations have been performed with one molecule of water as catalyst similar to that of JA-L-Ile. The carbonyl group of cis-Jasmonic acid-L-Leucine (3R,7S) (9) interacts with the water molecule to transfer the hydrogen from the C-7 carbon atom to the carbonyl group of cyclopentanone ring (Figure 4). The $\mathrm{C}=\mathrm{O}$ takes the hydrogen from the water molecule in the transition state (10) and the water takes the hydrogen from the C-7 atom. In the enol formation, the double bond of $\mathrm{C}=0$ breaks down and hydroxyl $\mathrm{OH}-$ group is formed at C-6 position leading to the formation of a double bond between C- 6 and C-7 atoms of cyclopentanone ring. As shown in JAL-Ile, the water molecule can deliver the hydrogen in two different ways: (i) the water molecule delivers the hydrogen from the syn-side of the leucine conjugated side chains and (ii) if the water molecule orients away from this side chain (Figure 4). We have examined both possibilities in this case too as we have done in the case of JA-L-Ile. The water molecule is hydrogen bonded to the enol-hydroxy group in both case (11 and 14, respectively). Further the water molecule can donate its hydrogen to the C-6 carbon centre and the hydroxy hydrogen can be taken by the same water molecule. The transition state geometries were obtained for both pathways (i) and (ii) (12 and 15, respectively).

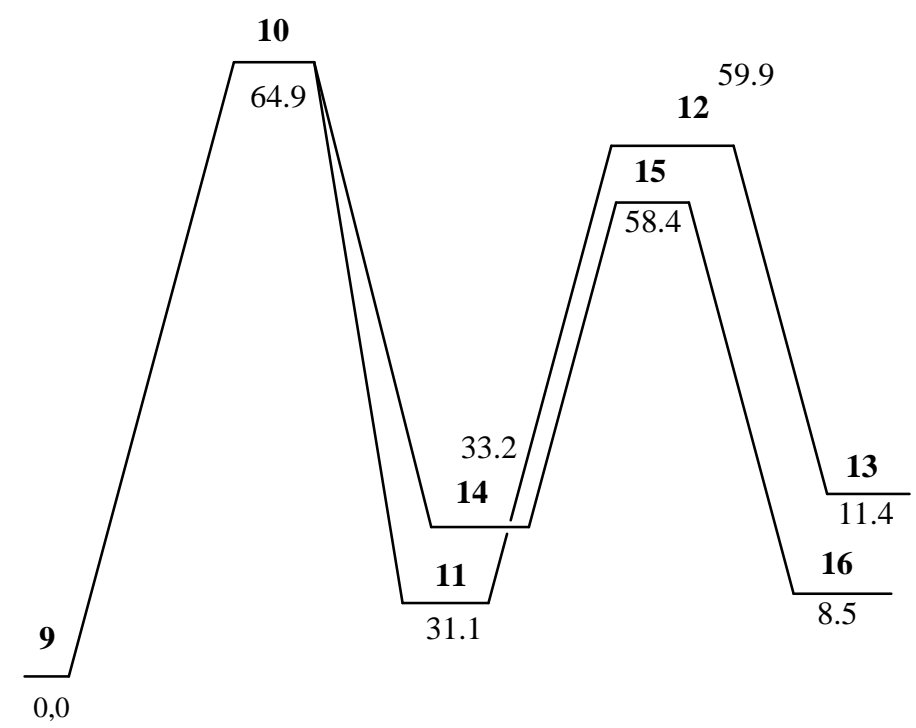

Figure 5. Potential energy surface profile for JA-L-Leu(3R,7S) in gas phase [All the energies are in $\mathrm{kcal} / \mathrm{mol}]$.

The potential energy profile for $(+) J A-L-L e u(3 R, 7 S)$ in the gas phase is shown in Figure 5. The activation barrier calculated for the formation of enol intermediate (11) is $64.9 \mathrm{kcal} / \mathrm{mol}$. The transition state structure (10) is similar to that of (2). The stability of two different enol-intermediates i.e.with the syn- sided $\mathrm{OH}(\mathbf{1 1})$ and the the $\mathrm{OH}$ antito the 2-pentenyl side chain (14) are 
similar in energy (Figure 5). The activation barriers calculated to form the cis- (13) and trans- $(\mathbf{1 6})$ products were found to be $59.9 \mathrm{kcal} / \mathrm{mol}$ and 58.8 $\mathrm{kcal} / \mathrm{mol}$, respectively. The PES profile from the B3LYP/6-31+G* level calculations shows that in a water catalyzed epimerization in JA-L-Leu (3R,7S), the trans-JA-L-Leu(3R,7R) (16) will be the favoured as stable product as we have seen in the case of JA-L-Ile.

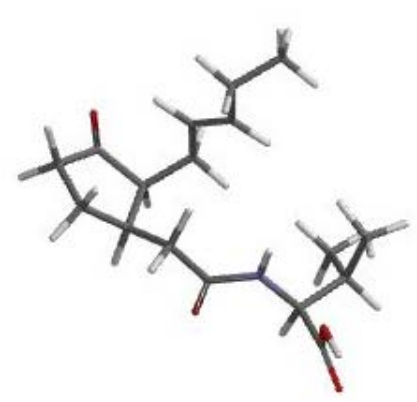

17

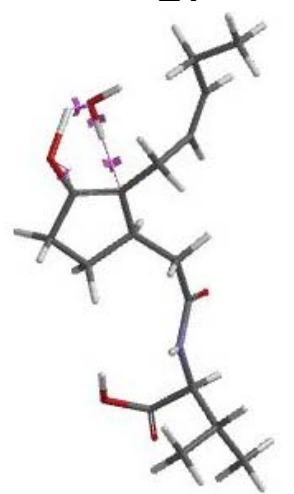

20

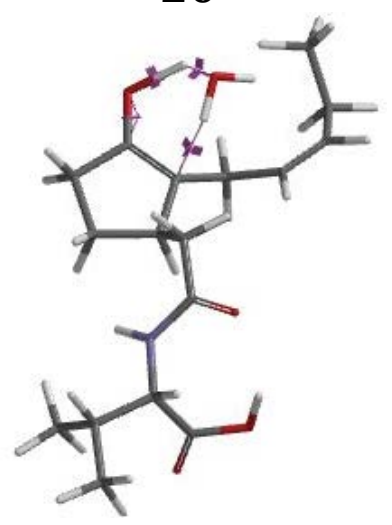

23

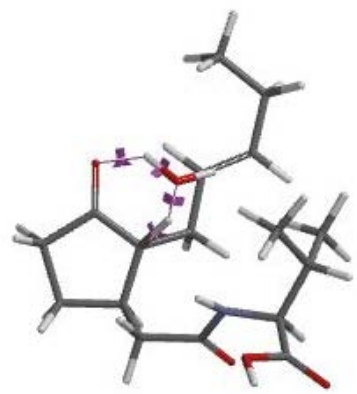

18

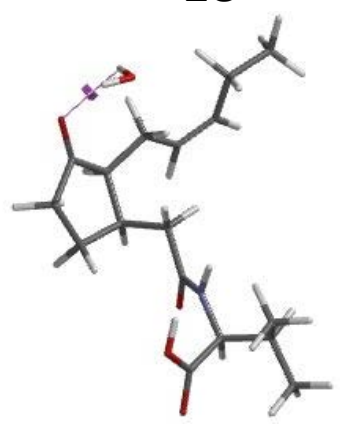

21

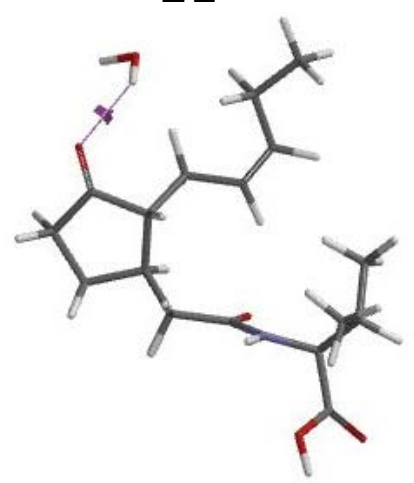

24

Figure 6. Geometry of JA-L-Val $(3 \mathrm{R}, 7 \mathrm{~S})$ obtained by optimization in AM1 Semi-empirical calculations. [17= Start $((+) J A-L-V a l(3 R, 7 S)), 18=$ Transition State, $19=$ Intermediate Enol form(syn-), 20= Enol-Transition State (i), 21 = Final (cis-(+)JA-L-Val(3R,7S))attached to water, 22 =Intermediate Enol form(anti), 23=Enol-Transition State (ii),24=Final(trans-(-)JA$\operatorname{Val}(3 R, 7 R))$ attached to water]n. 
The epimerization process in JA-L-Val $(3 R, 7 S)$ in presence of water molecule

We have also examined the epimerization of cis- or (+)JA -L$\operatorname{Val}(3 \mathrm{R}, 7 \mathrm{~S})$ to its trans-derivative, (-)JA-LVal(3R,7R) using AM1 and DFT studies (Figure 6).

The potential energy surface profile for cis- or (+)JA-L-Val $(3 R, 7 S)$ to its trans-derivative, (-)JA-L-Val(3R,7R) has been shown in Figure 7. The DFT calculated gas phase results show that the first epimerization transition state energy (18) is $59.8 \mathrm{kcal} / \mathrm{mol}$. Further, the other two transition states i.e., (20) and (23) are even higher than the first transition state (18) (Figure 7). The trans-derivative, (-)JA-L-Val(3R,7R) (21) is unstable compared to (17).

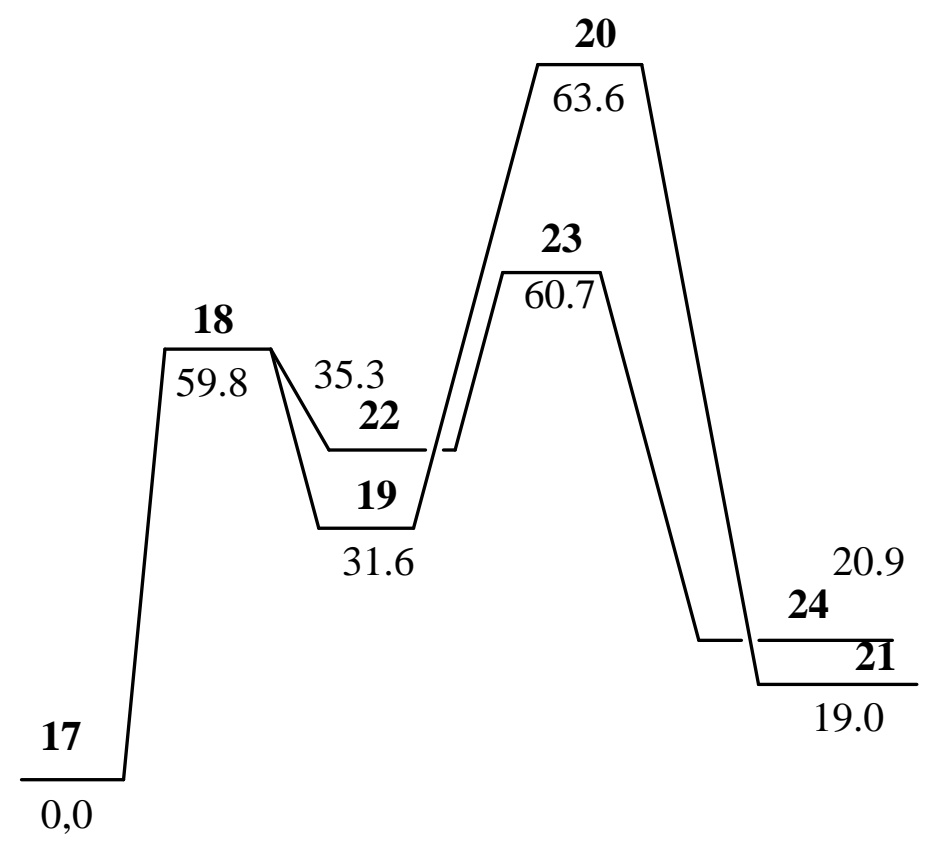

Figure 7. Potential energy surface profile for JA-L-Val(3R,7S) in gas phase [All the energies are in $\mathrm{kcal} / \mathrm{mol}]$.

To examine the influence of solvent on the potential energy surface of conjugation of Jasmonic acid with Isoleucine, Leucine and Valine, additional calculations were performed using PCM solvation model with B3LYP/6-31** level of theory. The aqueous phase calculations were performed using AM1 optimized geometries for all three amino acid conjugated Jasmonic acid. The calculated results show that the formation of enol is preferred with Jasmonic acid conjugated Isoleucine compared to Leucine and Valine. Nevertheless, the activation barriers were found to be relatively lower compared to the gas phase results in all cases (Figure 8, 9 and 10). 


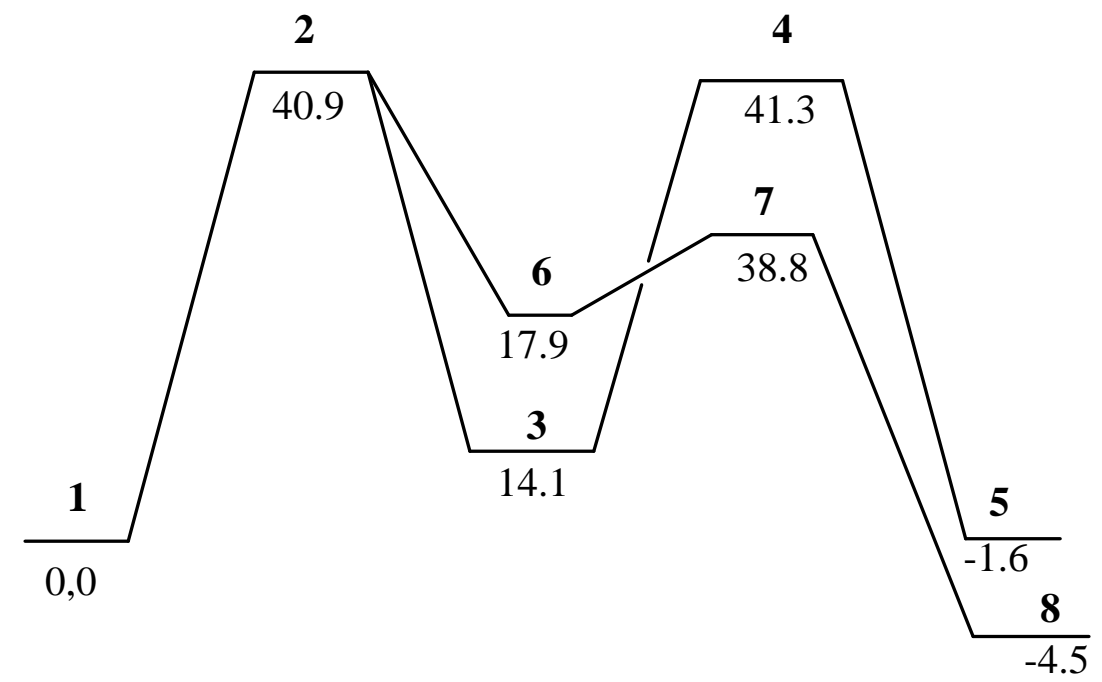

Figure 8. Potential energy surface profile for JA-L-Ile $(3 \mathrm{R}, 7 \mathrm{~S})$ in aqueous phase [All the energies are in $\mathrm{Kcal} / \mathrm{mol}]$

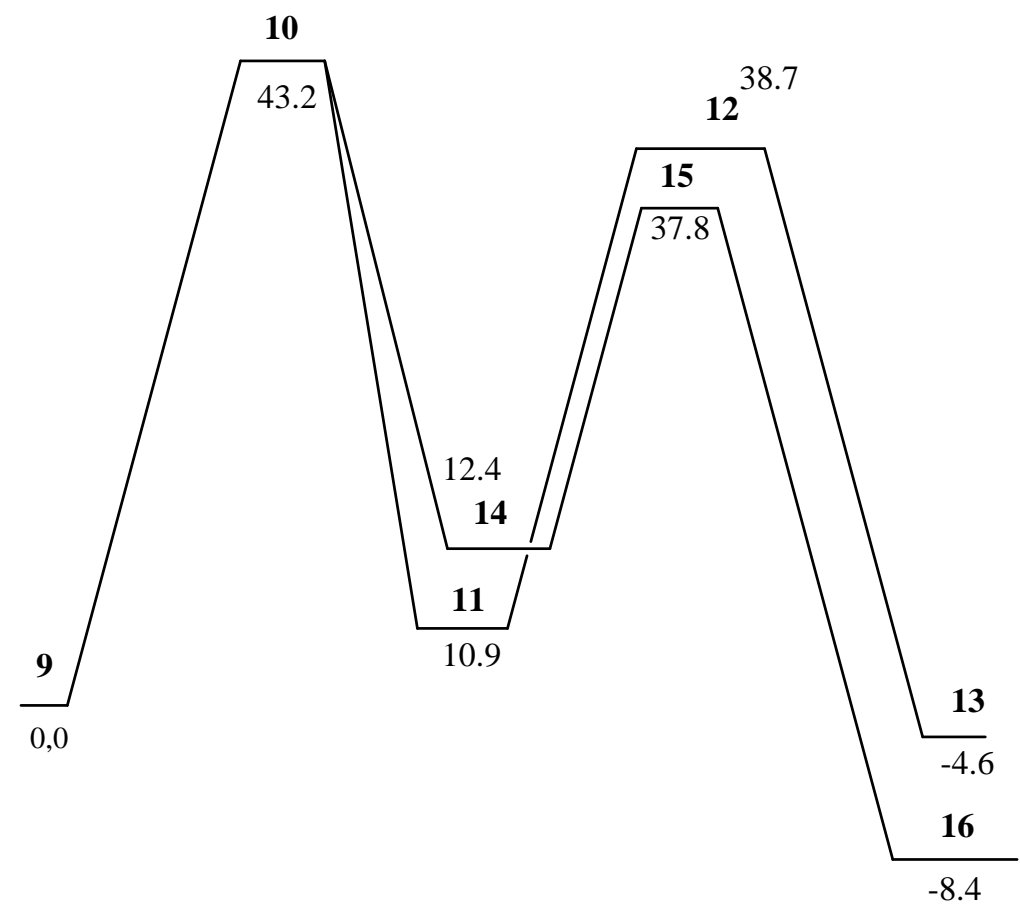

Figure 9. Potential energy surface profile for JA-L-Leu $(3 R, 7 S)$ in aqueous phase [All the energies are in $\mathrm{kcal} / \mathrm{mol}]$ 


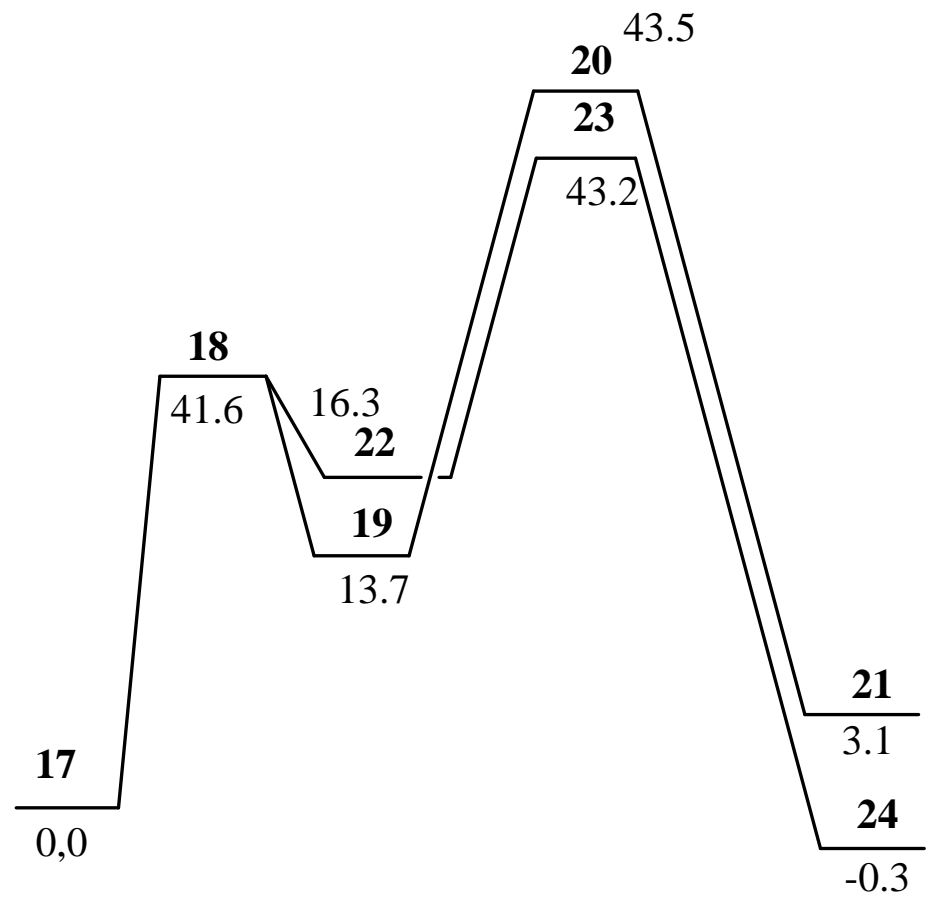

Figure 10. Potential energy surface profile for JA-L-Val $(3 R, 7 S)$ in aqueous phase [All the energies are in $\mathrm{kcal} / \mathrm{mol}]$.

The formation of enol with Jasmonic acids conjugated Valine is close to the formation of enol with Jasmonic acid conjugated Isoleucine, however, the formation of cis- and trans- products are energetically less favoured in the former case.

\section{Conclusions}

In this work, we have examined the role of amino acids in Jasmonate induced plant defense process. It has been found that conjugation of Jasmonic acid with Isoleucine is necessary to achieve such process effectively. Previous reports show that other Jasmonic acid-amino acid conjugates like JA-Leu and JA-Val do show some weak activity but only JA-Ile is recognised by the receptor COI1 and binds to it before binding to the JAZ protein in the JAsignalling process. Our computational work examined the origin of such process and showed that Jasmonic acidIsoleucine conjugate is more active compared to other two Jasmonic acid conjugates under consideration. Epimerisation process followed by enolization takes place in Jasmonic acidamino acid conjugates leading to a planar structure in upper half of the JA-amino acid conjugate before or after binding to the receptor molecule. This planarized form is suggested to be responsible for the effective binding with receptor molecule (Krumm et al., 1995). The calculated results revealed that the epimerization of (+)-7-iso-JA-LIle(3R,7S) to (-)JA-L-Ile(3R,7R) energetically is a favoured process compared to JA-Leu and JA-Val. The B3LYP/6-31+G*//RHF/AM1 level of theory calculations suggests that calculated activation barrier for the epimerization process of JA-Isoleucine is $45.0 \mathrm{kcal} / \mathrm{mol}$, whereas such barriers were found to be $\sim 60.0 \mathrm{kcal} / \mathrm{mol}$ for JALeu and JA-Val. . Importantly, the energy difference between the JA-L-Ile(3R,7S) and JA-L-Ile(3R,7R) isomers too are relatively smaller compared to the corresponding Leu and Val isomers. According to our computed results the 
formation of $(+)$ or the $(-)$ forms of JAIsoleucine is thermodynamically stable in the potential energy surface. Therefore, the chances of availability of cis- and trans-isomers of Isoleucine conjugated JA in the plants to defend the wound attack process. The computed results suggest that the either the presence of JA-L-Ile(3R,7S) to JA-LIle(3R,7R) due to their small energy difference can influence the plant defense process as mentioned in the different reports (Wasternack C, 2010; Krumm,T. et al., 1995). The aqueous phase calculations also corroborated the gas phase results.

In the whole epimerisation process water has functioned as a catalyst which is in abundance in any plant. Further, the discussion of planarity of the intermediate is also observed in all the three cases. Nevertheless, the overall epimerization of Leucine and Valine conjugated Jasmonic acids are least likely and hence the presence of JA-LLue(3R,7S) and JA-L-Val(3R,7S) is least likely in this process. Therefore, we may conclude that the presence of JA-LLue(3R,7S) and JA-L-Val(3R,7S) may be necessary for their effectiveness in the plant defense process, which however, is not observed computationally in the present study. These results further indicate that the Isoleucine conjugated Jasmonic acid is more effective in the plant defense mechanism compared to Leucine and Valine conjugated Jasmonic acids.

\section{Conflict of interest}

The authors declare that they have no conflict of interest in the publication.

\section{Acknowledgement}

Ruma Ganguly thanks Sir P. P. Institute of Science for all the necessary help to carry out this work.

\section{Reference}

Becke, A. D. Density functional thermochemistry. II. The effect of the Perdew-Wang generalized-gradient correlation correction. Journal of Chemical Physics, v. 97, p. $9173, \quad 1992$. https://doi.org/10.1063/1.463343

Browse, J.; Howe, G. A. New weapons and a rapid response against insect attack. Plant Physiology, v. 146, p. 832-838, 2008. https://doi.org/10.1104/pp.107.115683

Clark, T. A handbook of computational chemistry. New York: Wiley Interscience, 1985.

Cramer, C. J.; Truhlar, D. G. AM1-SM2 and PM3-SM3 parameterized SCF solvation models for free energies in aqueous solution. Journal of Computer-Aided Molecular Design, v. 6, no. 6, p. 629-666, 1992.

Dewar, M. S.; Zoebisch, E. G.; Healy, E. F.; Stewart, J. J. The development and use of quantum mechanical molecular models. 76. AMI: A new general purpose quantum mechanical molecular model. Journal of American Chemical Society, v.107, p. 3902-3909, 1985.

Feussner I.; Wasternack,C., The lipoxygenase pathway. Annual Review in Plant Biology, v. 53, p. 275-297, 2002. https://doi.org/ 10.1146/annurev.arplant.53.100301.135248

Fonseca, S.; Chini, A.; Hamberg, M.; Adie, B.; Porzel, A.; Kramell, R.; Miersch, O.; Wasternack, C.; Solano, R. (+)-7-isoJasmonoyl-L-isoleucine is the endogenous bioactive jasmonate. Nature Chemical Biology, v. $5, \quad$ p. 344-350, 2009. https://doi.org/10.1038/nchembio.161

Gundlach, H.; Müller, M. J.; Kutchan, T. M.; Zenk, M. H. Jasmonic acid is a signal transducer in elicitor-induced plant cell cultures. Proceedings of the National Academy of Science of the U.S.A., v. 89, no. 6, p. 2389-2393, 1992.

Hehre, W. J.; Radom, L.; Schleyer, P. V. R.; Pople, J. A. Ab initio molecular orbital theory. New York: John Wiley \& Sons, 1986.

Holbrook, L.; Tung, P.; Ward, K.; Reid, D. M.; Abrams, S.; Lamb, N.; Quail, J. W.; and Moloney, M. M. Importance of the chiral centers of jasmonic acid in the responses of plants-activities and antagonism between natural and synthetic analogs. Plant Physiology, v. 114, no. 2, p. 419-428, 1997. 
Koo, A. J. K.; Howe, G. A. The wound hormone jasmonate. Phytochemistry, v. 70, p. 15711580, 2009. https://doi.org/10.1016/ j.phytochem.2009.07.018

Krumm, T.; Bandemer, K.; Boland, W. Induction of volatile biosynthesis in the lima bean (Phaseolus lunatus) by leucine- and isoleucine conjugates of 1-oxo- and 1-hydroxyindan-4-carboxylic acid - evidence for amino acid conjugates of jasmonic acid as intermediates in the octadecanoid signalling pathway. FEBS Letters, v. 377, no. 3, p. 523529, 1995. https://doi.org/10.1016/00145793(95)01398-9

Lee, C.; Yang, W.; Parr, R. G. Development of the Colle-Salvetti correlation-energy formula into a functional of the electron density. Physical Review B, v. 7, no. 2, p. 785-789, 1988. https://doi.org/10.1103/PhysRevB. 37.785

McConn, M.; Creelman, R.A.; Bell, E.; Mullet, J.E.; Browse, J. Jasmonate is essential for insect defense in Arabidopsis. Proceedings of the National Academy of Science of the U.S.A., v. 94, no. 7, 5473-5477, 1997.

Miersch O.; Kramell R.; Parthier, B.; Wasternack, C. Structure-activity relations of substituted, deleted or stereospecifically altered jasmonic acid in gene expression of barley leaves. Phytochemistry, v. 50, p. 353-361, 1999.

Naoki, K.; Hiroshi, K.; Naruki, A.; Takuya, M.; Kensuke, N.; Kosaku, T.; Hideyukiv, M.; CYP94B3 activity against asmonic acid amino acid conjugates and the elucidation of
12-0- $\beta$-glucopyranosyl-jasmonoyl-Lisoleucine as an additional metabolite. Phytochemistry, v. 99, p.6-13, 2014. https://doi.org/10.1016/j.phytochem.2013.1 2.019

Piotrowska, A.; Bajguz, A.; Conjugates of abscisic acid, brassinosteroids, ethylene, gibberellins, and jasmonates. Phytochemistry, v. 72, p. 2097-2112, 2011. https://doi.org/10.1016/j.phytochem.2011.0 8.012

Ryan, C.A. The search for the proteinase inhibitor-inducing factor, PIIP. Plant Molecular. Biology, v. 19, p. 123-133, 1992.

Stintzi, A.; Weber, H.; Reymond, P.; Browse, J.; Farmer; E.E. Plant defense in the absence of jasmonic acid: The role of cyclopentanones. Proceedings of the National Academy of Science of the U.S.A., v. 98, p. 12837-12842, 2002.

Vick, B.; Zimmerman, D. C. Biosynthesis of jasmonic acid by several plant species. Plant Physiology, v. 75, p. 458-461, 1984.

Wasternack, C. Jasmonates: An update on biosynthesis, signal transduction and action in plant stress response, growth and development. Annals of Botany, v. 100, no. 4, p. 681-697, 2007. https://doi.org/ 10.1093/aob/mcm079

Wasternack, C.; Kombrink, E. Jasmonates: Structural requirements for lipid-derived signals active in plant stress responses and development. ACS Chemical Biology, v. 5, p. 63-77, 2010. https://doi.org/10.1021/ cb900269u 